\title{
VECTOR FIELDS ON SPHERES
}

\author{
BY HIROSI TODA
}

Communicated by Deane Montgomery, March 27, 1961

1. The problem is to determine the maximal number of the independent continuous fields of tangent vectors on the unit $n$-sphere $S^{n}$. The number will be denoted by $\lambda(n)$.

$\lambda(n)$ is the maximal number of $k$ such that the boundary homomorphism $\Delta_{n, k}: \pi_{n}\left(S^{n}\right) \rightarrow \pi_{n-1}\left(O_{n, k}\right)$ associated with the fibering $O_{n+1, k+1} / O_{n, k}=S^{n}$ is trivial, where $O_{n, k}$ denotes the Stiefel manifold of the orthogonal $k$-vectors ( $k$-frames) in the real $n$-space $R^{n}$.

The fundamental conjecture for our problem is stated as follows.

ConJeCtuRe. Does $\lambda(n)=\lambda^{*}(n)$ for all $n>0$ ?

Here, the conjectured values $\lambda^{*}(n)$ are defined as follows:

$$
\begin{aligned}
\lambda^{*}(n) & =\lambda_{r}, \quad \text { if } n \equiv 2^{r}-1\left(\bmod 2^{r+1}\right), \\
\lambda_{0} & =0, \quad \lambda_{1}=1, \quad \lambda_{2}=3, \quad \lambda_{3}=7
\end{aligned}
$$

and

$$
\lambda_{r+4}=\lambda_{r}+8 .
$$
[4].

It was known that the conjecture is true for the cases $r=0,1,2,3$

The obtained results on $\lambda(n)$ are the following.

THEOREM 1. (a) $\lambda^{*}(n) \leqq \lambda(n)$. (b) If $k=\lambda^{*}(n)$, then the image of $\Delta_{n, k}: \pi_{n}\left(S^{n}\right) \rightarrow \pi_{n-1}\left(O_{n, k}\right)$ coincides with the image of the composition $i_{*} \circ J: \pi_{k}(S O(n-k-1)) \rightarrow \pi_{n-1}\left(S^{n-k-1}\right) \rightarrow \pi_{n-1}\left(O_{n, k}\right)$ of $G$. Whitehead's homomorphism $J$ and the homomorphism $i_{*}$ induced by the usual injection $i: S^{n-k-1} \subset O_{n, k}$.

The first part (a) is provided by the recent work of Bott and Shapiro, Clifford modules and vector fields on spheres (mimeographed note), which states the existence of a continuous field of linear $\lambda^{*}(n)$-frames on $S^{n}$.

Theorem 2. $\lambda^{*}(n)=\lambda(n)$ if $n \equiv 2^{r}-1\left(\bmod 2^{r+1}\right)$ for an integer $r<11$.

Then our problem is still open in question on the sphere $S^{2047}$.

Theorem 3. $\lambda\left(2^{i} m-1\right) \geqq \lambda(m-1)+2^{i-1}$ for $i=1,2,3,4$.

CoRollary. If the above conjecture is not true for an $n \equiv 2^{r}-1$ $\left(\bmod 2^{r+1}\right)$ and $r=4 s-1(s:$ positive integer), then the conjecture is not true for all $n$ of $r \geqq 4 s-1$. 
2. The following lemma means that our problem is a stable one, that is, we may assume that for each $r$ the integer $n$ is sufficiently large.

LeMma 1. $\lambda^{*}\left(2^{r}-1\right) \leqq \lambda\left(2^{r}-1\right)$ if and only if $\lambda^{*}(n) \leqq \lambda(n)$ for an integer $n$ of $n \equiv 2^{r}-1\left(\bmod 2^{r+1}\right)$.

This lemma is proved by applying the theory [3] of James.

In the following, we always assume that the integers $n$ are sufficiently large with respect to the other integers $k$ and $i$ such that the homology and homotopy considered are stable. Then we can replace $O_{n, k}$ by a cell complex

$$
P_{n, k}=P^{n-1} / P^{n-k-1}=S^{n-k} \cup e^{n-k+1} \cup \cdots \cup e^{n-1}
$$

which is obtained from real projective $(n-1)$-space $P^{n-1}$ by shrinking its $(n-k-1)$-subspace $P^{n-k-1}$ to a point, since the cellular decomposition of $O_{n, k}$ given in [7] shows that $P_{n, k}$ is a subcomplex of $O_{n, k}$ and the dimensionalities of $O_{n, k}-P_{n, k}$ are greater than $2 n-2 k$. The exact sequence for the fibering $O_{n+1, k+1} / O_{n, k}=S^{n}$ is replaced by the following exact sequence:

$$
\cdots \rightarrow \pi_{n}\left(S^{n}\right) \stackrel{\Delta_{n, k}}{\longrightarrow} \pi_{n-1}\left(P_{n, k}\right) \stackrel{i_{*}}{\rightarrow} \pi_{n-1}\left(P_{n+1, k+1}\right) \rightarrow \cdots
$$

Now our problem is transformed to a problem on the homotopy of $P_{n+1, k+1}$.

LEMMA 2. $\lambda(n)$ is a maximal number of $k$ such that the attaching map of the $n$-cell $e^{n}=P_{n+1, k+1}-P_{n, k}$ is inessential in $P_{n, k}$, namely, $\Delta_{n, k}\left(\iota_{n}\right)=0$ for the class $\iota_{n} \in \pi_{n}\left(S^{n}\right)$ of the identity of $S^{n}$.

The following two lemmas are obtained by translating results of [3] in our words.

Lemma 3. If $k \leqq \lambda(m-1)$, then the $m$-fold iterated suspension $E^{m} P_{n, k+1}$ of $P_{n, k+1}$ and $P_{n+m, k+1}$ have the same homotopy type.

In fact, a homotopy equivalence is given by the composition of the join: $E^{m} P_{n, k+1}=P_{n, k+1} * S^{m-1} \rightarrow P_{n, k+1} * P_{m, k+1}$ of the identity and a cross-section: $S^{m-1} \rightarrow P_{m, k+1}$ with the intrinsic join: $P_{n, k+1} * P_{m, k+1}$ $\rightarrow P_{m+n, k+1}$.

Lemma 4 . Let $n$ be odd, $h \leqq k \leqq \lambda(n)$ and $n$ be large $(n \geqq 2(k+h))$. Assume that $\Delta_{n, k+h}\left(\iota_{n}\right)$ is the image of $\alpha \in \pi_{n-1}\left(P_{n-k, h}\right)$ under the homomorphism $i_{*}$ induced by the injection $i: P_{n-k, h} \subset P_{n, k+h}$, then $\Delta_{2 n+1, k+h}\left(\iota_{2 n+1}\right)$ is the image of $2 E^{n+1} \alpha \in \pi_{2 n}\left(P_{2 n+1-k, h}\right)$ under the homomorphism $i_{*}^{\prime}$ induced by the injection $i^{\prime}: P_{2 n+1-k, h} \subset P_{2 n+1, k+h}$. 
Briefly stated, $\Delta_{n, k+h}\left(\iota_{n}\right)$ is an obstruction to the inequality $k+h$ $\leqq \lambda(n)$ and two times it is an obstruction to $k+h \leqq \lambda(2 n+1)$, since $E^{n+1}$ is an isomorphism.

3. Let $K_{n, k}$ be a simply connected finite cell complex having the same homology of $P_{n, k}$. As $n$ is so large, then $K_{n, k}$ has the same homotopy type as a suspension of a complex. The set of all the homotopy classes of the mappings of $K_{n, k}$ in itself forms a group as in [1]. Let $\iota_{n, k}$ be the class of the identity of $K_{n, k}$.

Lemma 5. Let $n$ be odd and large $(n>4 k)$. Then $2^{t_{t_{n, 2}}}=0$ for $\lambda_{t-1}$ $\geqq 2 k-1$. For example, $4 \iota_{n, 2}=0,8 \iota_{n, 4}=0$ and $16 \iota_{n, 6}=16 \iota_{n, 8}=0$.

This is proved by giving deformations in $K_{n, 2 k}$ and by applying the results on the stable groups $\pi_{n+k}\left(S^{n}\right)$ for $n \leqq 7$.

Corollary. Let $n$ be odd and large with respect to $k$ and $i$. Then $2^{t} \pi_{n+i}\left(K_{n, 2 k}\right)=0$ and $2^{t} \pi^{n+i}\left(K_{n, 2 k}\right)=0$ for $\lambda_{t-1} \geqq 2 k-1$, where $\pi^{n+i} d e-$ notes the $(n+i)$ th cohomotopy group.

Now Theorem 3 is a consequence of this corollary and Lemma 4. As another application of this corollary, we have the following:

THEOREM 4. Let $n$ be odd and $\lambda_{t-1} \geqq 2 k-1$. Let $\Omega^{2 k}\left(S^{n+2 k}\right)$ be the $2 k$-fold iterated loop-space of $S^{n+2 k}$. Then $2^{t} \pi_{i}\left(\Omega^{2 k}\left(S^{n+2 k}\right), S^{n}\right)=0$ for $i \leqq 3 n-2$. If $i \leqq 4 n-3$, then $2^{t} \pi_{i}\left(\Omega^{2 k}\left(S^{n+2 k}\right), S^{n}\right)$ has no 2-torsion.

By a similar method to $J$-homomorphism [6] of G. Whitehead, we have a homomorphism $J: \pi_{i-1}\left(E^{n-1} P_{n+2 k, 2 k}\right) \rightarrow \pi_{i}\left(\Omega^{2 k}\left(S^{n+2 k}\right), S^{n}\right)$, which is an isomorphism if $i \leqq 3 n-2$ and an isomorphism of the 2primary components if $i \leqq 4 n-3$. Then the theorem is proved by the above corollary.

As a corollary of Theorem 4 , we have similar statements for the kernel and cokernel of $E^{2 k}: \pi_{i}\left(S^{n}\right) \rightarrow \pi_{i+2 k}\left(S^{n+2 k}\right)$.

4. Denote by $J_{k} \subset \pi_{n+k}\left(S^{n}\right)$ the image of G. Whitehead's $J$-homomorphism $J: \pi_{k}(S O(n)) \rightarrow \pi_{n+k}\left(S^{n}\right)$. Applying Bott's periodicity $\Omega^{8} S O(\infty)=S O(\infty)$, the following lemma is proved.

Lemma 6. Let $n$ be large. Let $\eta$ be the generator of $J_{1}$, and let $\sigma^{h}$ and $\zeta^{h}$ be generators of $J_{8 h-1}$ and $J_{8 h+3}$ respectively, $h=1,2, \cdots$. Then $J_{8 h}$ and $J_{8 h+1}$ are generated by the compositions $\sigma^{h} \circ \eta$ and $\sigma^{h} \circ \eta \circ \eta$, respectively, and $\zeta^{h}$ and $\sigma^{h+1}$ are represented by compositions $g \circ f: S^{n+8 h+3}$ $\rightarrow S^{n+8 h-1} \cup e^{n+8 h+8} \rightarrow S^{n}$ and $g^{\prime} \circ f^{\prime}: S^{n+8 h+r} \rightarrow S^{n+8 h-1} \cup e^{n+8 h+7} \rightarrow S^{n}$, respectively, where $g \mid S^{n+8 h-1}$ and $g^{\prime} \mid S^{n+8 h-1}$ represent $\sigma^{h}, f$ and $f^{\prime}$ induce 
homomorphisms of degree 2 of the homology groups and the cells $e^{n+8 h+8}$ and $e^{n+8 h+7}$ are attached to $S^{n+8 h-1}$ by essential mappings.

The proof of Theorem 1 is done by induction on $r$. Assume that Theorem 1 is proved for an $r=4 h-1, h \geqq 1$. In this case, $P_{n-8 h-1,3}$ is of the same homotopy type as $P_{n-8 h-2,2} \vee S^{n-8 h-2}$ and $\Delta_{n, 8 h+2}\left(\iota_{n}\right)$ $=i_{*}\left(\beta^{\prime}+\sigma^{h}\right)$ for $\beta^{\prime} \in \pi_{n-1}\left(P_{n-8 h-2,2}\right)$ and $\sigma^{h} \in \pi_{n-1}\left(S^{n-8 h-2}\right)$. In the complex $P_{n-8 h-2,2}=S^{n-8 h-4} \cup e^{n-8 h-3}$ the cell $e^{n-8 h-3}$ is attached by degree 2 . Then by shrinking $S^{n-8 h-4}$ to a point, the element $\beta^{\prime}$ goes to an element $\beta \in \pi_{n-1}\left(S^{n-8 h-3}\right)$ such that $2 \beta=0$. Thus $\Delta_{n, 8 h+1}\left(\iota_{n}\right)=i_{*}\left(\beta+\sigma^{h}\right)$ for $2 \beta=0$. Consider $2 i_{*}\left(\beta+\sigma^{h}\right)=i_{*}\left(2 \sigma^{h}\right)$. Since the cell $e^{n-8 h-1}=P_{n-8 h, 3}$ $-P_{n-8 h-1,2}$ is attached to $P_{n-8 h-1,2}=S^{n-8 h-3} \bigvee S^{n-8 h-2}$ by a mapping which represents $\eta$ at $S^{n-8 h-3}$ and is of degree 2 at $S^{n-8 h-2}$, it follows that $2 \sigma^{h} \in \pi_{n-1}\left(S^{n-8 h-2}\right)$ and $\sigma^{h} \circ \eta \in \pi_{n-1}\left(S^{n-8 h-3}\right)$ go to the same element by the injections into $P_{n-8 h, 3}$. By Lemma 4 , we have that $\Delta_{2 n+1,8 h+1}\left(\iota_{2 n+1}\right)=i_{*} E^{n+1}\left(\sigma^{h} \circ \eta\right)$ and this is the statement of Theorem 1 for $r=4 h$.

Next step of the proof is done by showing that if $\Delta_{n, 8 h+2}\left(\iota_{n}\right)=i_{*} \alpha$ for an element $\alpha \in \pi_{n-1}\left(P_{n-8 h-2,2}\right)$ which goes to $\sigma^{h} \circ \eta$ by shrinking $S^{n-8 h-4}$, then $\sigma^{h} \circ \eta \circ \eta \in \pi_{n-1}\left(S^{n-8 h-4}\right)$ goes to $2 \alpha$ by the injection of $S^{n-8 h-4}$ into $P_{n-8 h-2,2}$.

The remaining two steps of the proof of Theorem 1 are too complicated to describe here, but it can be done by applying above lemmas.

5. The proofs of Theorem 2 are purely computations of the homotopy groups $\pi_{n-1}\left(P_{n, k}\right)$ for $k=\lambda^{*}(n)+1$, by showing that $\Delta_{n, k}\left(\iota_{n}\right) \neq 0$. In the computations, the following results on the homotopy groups of spheres and several relations in them are used [5]. Let $\left(G_{k} ; 2\right)$ be the 2-primary component of the stable group $\pi_{n+k}\left(S^{n}\right)$, $n>k+1$; then we have the following table.

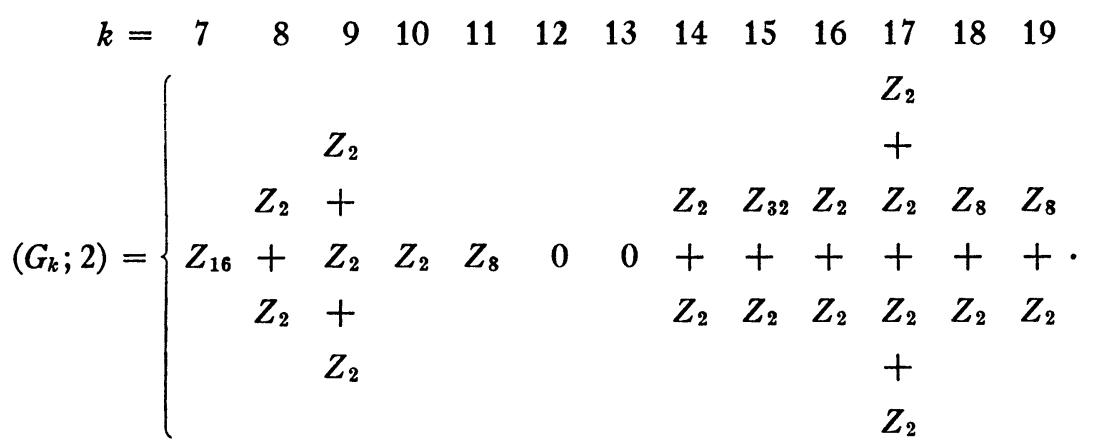




\title{
BIBLIOGRAPHY
} 106.

1. M. G. Barratt, Truck groups. I, Proc. London Math. Soc. vol. 5 (1955) pp. 71-

2. R. Bott, The stable homotopy of the classical groups, Ann. of Math. vol. 70 (1959) pp. 313-337.

3. I. M. James, The intrinsic join: a study of the homotopy groups of Stiefel manifolds, and Cross-sections of Stiefel manifolds, Proc. London Math. Soc. vol. 8 (1958) pp. 507-636 and pp. 536-547.

4. - Whitehead products and vector fields on spheres, Proc. Cambridge Philos. Soc. vol. 53 (1957) pp. 817-820.

5. H. Toda, Composition methods in homotopy groups of spheres, to be published in Annals of Mathematics Studies, Princeton University.

6. G. W. Whitehead, A generalization of the Hopf invariant, Ann. of Math. vol. 51 (1950) pp. 192-237.

7. J. H. C. Whitehead, On the groups $\pi_{r}\left(V_{n, m}\right)$ and sphere-bundles, Proc. London Math. Soc. vol. 48 (1944) pp. 243-291.

The Institute for Advanced Study and UNIVERSITY OF KYOTO

\section{COMPACT KAEHLER MANIFOLDS WITH POSITIVE RICCI TENSOR}

\author{
BY SHOSHICHI KOBAYASHI
}

Communicated by I. M. Singer, March 6, 1961

The purpose of the present note is to announce the following:

Theorem 1. A compact Kaehler manifold with positive definite Ricci tensor is simply connected.

We say that the first Chern class of a compact Kaehler manifold is positive definite if it can be represented by a real closed $(1,1)$-form which is positive in the sense of Kodaira [2]. The first Chern class of a manifold satisfying the assumption in Theorem 1 is necessarily positive definite. Theorem 1 follows from the following two theorems.

THEOREM 2. If the first Chern class of a compact Kaehler manifold $M$ is positive definite, then the fundamental group of $M$ has no proper subgroup of finite index.

THEOREM OF Myers. The fundamental group of a compact Riemannian manifold with positive definite Ricci tensor is finite [3].

Theorem 2 can be proved by Kodaira's Vanishing Theorem and by the Riemann-Roch Theorem of Hirzebruch. Let $g_{p}$ be the dimension of the space of holomorphic $p$-forms on $M$. Then $\chi(M)=\sum_{p=0}^{n}(-1)^{p} g_{p}$, where $n=\operatorname{dim}_{C} M$, is called the arithmetic genus of $M$. If $M$ is 